\title{
Case of the month: What's your diagnosis?
}

Peterhans, Sophie ; Ghielmetti, Giovanni ; Botta, Carolina ; Friedel, Ute ; Hilbe, Monika ;

Schneeberger, M ; Stephan, Roger

DOI: https://doi.org/10.17236/sat00185

Posted at the Zurich Open Repository and Archive, University of Zurich ZORA URL: https://doi.org/10.5167/uzh-168129

Journal Article

Published Version

Originally published at:

Peterhans, Sophie; Ghielmetti, Giovanni; Botta, Carolina; Friedel, Ute; Hilbe, Monika; Schneeberger, M; Stephan, Roger (2018). Case of the month: What's your diagnosis? Schweizer Archiv für Tierheilkunde, 160(11):673-675.

DOI: https://doi.org/10.17236/sat00185 


\section{Case of the month: What's your diagnosis?}

https://doi.org/ $10.17236 /$ sat00185

Received: 05.07.2018 Accepted: 24.09.2018

\author{
S. Peterhans'1, G. Ghielmetti', C. Botta², U. Friedel'1, M. Hilbe'2, \\ M. Schneeberger ${ }^{1}$, R. Stephan ${ }^{1}$
}

${ }^{1}$ Institute of Veterinary Bacteriology and ${ }^{2}$ Institute of Veterinary Pathology, Vetsuisse Faculty, University of Zurich, Switzerland

\begin{abstract}
Animal and gross pathological examination A European brown hare (Lepuseuropaeus) kept in a small group (5-8 animals) in a wildlife park in Zurich, died peracutely without any premonitory clinical signs. No other in-contact animals (hares, brown rats (Rattus norvegicus) and house mice (Mus musculus)) showed any clinical signs. A full necropsy of the dead hare was performed at the Institute of Veterinary Pathology of the University of Zurich. The animal was in poor body condition (emaciation) as indicated by the lack of intraabdominal, perirenal and pericardial fat tissue depots. No other gross findings were detected.
\end{abstract}

\section{Histopathological examination}

Representative tissue samples from multiple organs were fixed in 4\% formalin and paraffin-embedded for histological examination. 2-3 $\mu \mathrm{m}$ sections were prepared and stained with haematoxylin and eosin ( $\mathrm{H} \& \mathrm{E})$ according to standard procedures. Histopathology of the liver revealed multifocal, randomly distributed, small foci of necrotic hepatocytes showing hypereosinophilic cytoplasm and pyknotic nuclei accompanied by moderate amounts of degenerated neutrophils and macrophages (Fig. 1A). Multifocally to coalescing, in the red pulp of the spleen, small foci of fibrinous exsudate and necrosis (Fig. 1B) were observed. The mesenteric lymph nodes showed multifocal to diffuse necrosis and infiltrates of degenerated neutrophils and macrophages (Fig. 1C).

\section{Microbiological examinations}

A heart blood sample, taken at necropsy, was cultured on chocolate agar (Oxoid, Pratteln, Switzerland) and incubated at $37^{\circ} \mathrm{C}$ under an atmosphere of $5 \% \mathrm{CO}_{2}$ for 96 hours. Bacterial growth occurred and thereafter, all further examinations were performed at the BSL-3 laboratory containment. Gram staining showed gram-negative small coccobacilli. Catalase and oxidase reaction tested negative.

Formulate your differential diagnoses from the history, histopathology, microbiological examinations, and Figure 1. How would you confirm it? - then turn the page $\rightarrow$

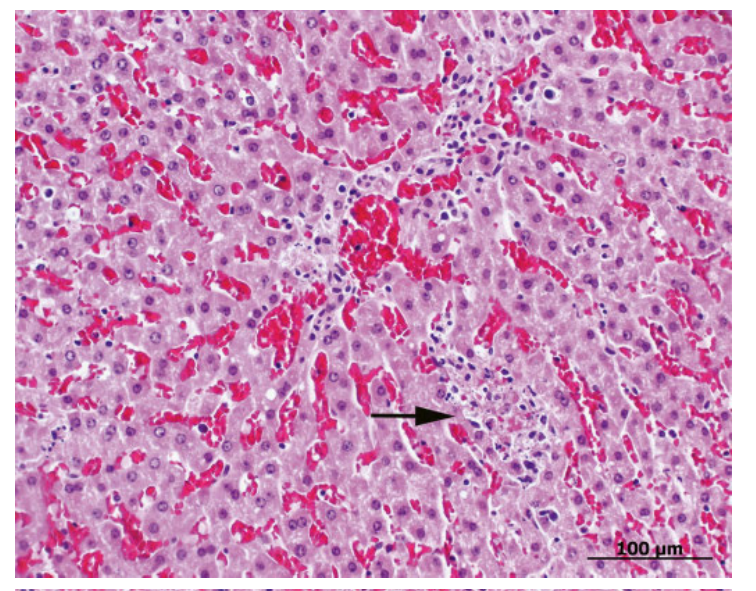

Figure 1A: Histopathology of liver in an European brown hare (Lepus europaeus). Illustrating necrotic hepatocytes accompanied by infiltrates of degenerated neutrophils and macrophages (arrow; 200× magnification, H\&E).

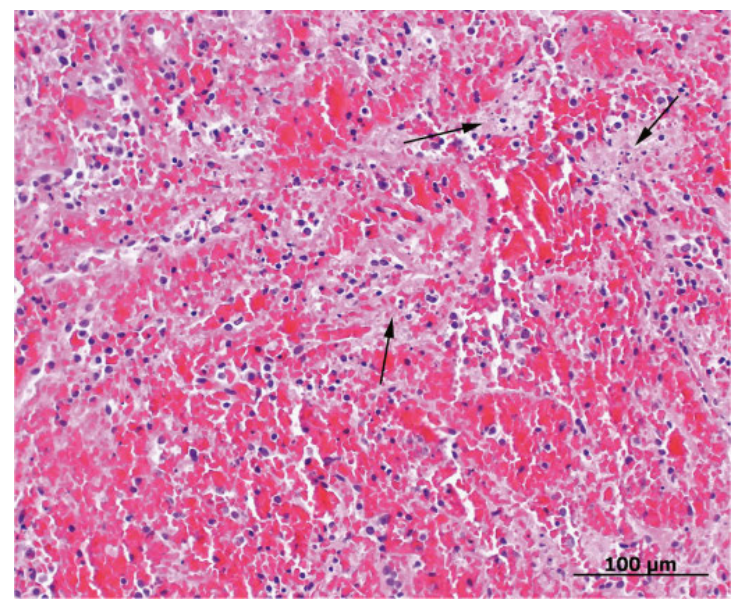

Figure 1B: Histopathology of the spleen in an European brown hare (Lepus europaeus). Foci of fibrinous exudate and necrosis in the red pulp of the spleen are visible (arrow; 200× magnification, $\mathrm{H} \& \mathrm{E}$ )

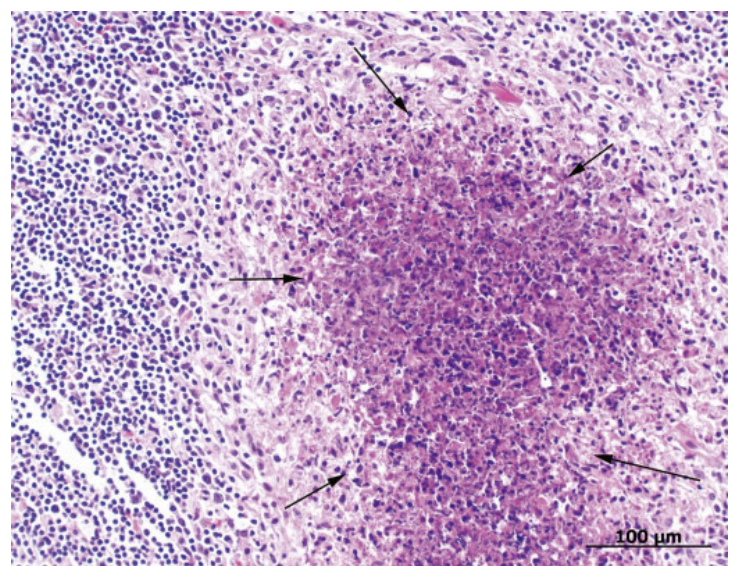

Figure 1C: Histopathology of the mesenteric lymphnode in an European brown hare (Lepus europaeus). The mesenteric lymph node shows diffuse necrosis and infiltrates of degenerated neutrophils and macrophages (arrow; 40x magnification, $\mathrm{H} \& \mathrm{E})$. 


\section{Case of the month: What's your diagnosis? \\ Further examinations}

S. Peterhans et al.

\section{PCR diagnosis, subtyping of Francisella tularensis and erythromycin susceptibility testing}

The presumptive result "Francisella spp." was confirmed by PCR at the reference laboratory (ZOBA).

For subtyping, DNA was isolated using InstaGene Matrix (Bio Rad, Cressier, Switzerland) according to the standard protocol (heated twice at $56^{\circ} \mathrm{C}$ for $30 \mathrm{~min}$ and at $100^{\circ} \mathrm{C}$ for $30 \mathrm{~min}$, subsequently centrifuged at $12^{\prime} 000$ $\mathrm{rpm}$ for $3 \mathrm{~min})$. DNA concentration of the supernatant was measured by reading the absorbance at $260 \mathrm{~nm}$. The PCR for subtyping of Francisella (F.) tularensis was performed with the method described by Abril et al. 20071 . Based on the fragment size of the PCR amplicon, the two F. tularensis subspecies can be distinguished into bolarctica (1522bp) and tularensis (924bp) 1 . With an amplicon length of $1522 \mathrm{bp}$, the isolate was identified as $F$. tularensis ssp. holarctica. To further distinguish between Biovar I (erythromycin susceptible) and II (erythromycin resistant) susceptibility testing was performed using the disc-diffusion method (15 $\mu \mathrm{g} / \mathrm{disc}$, Oxoid) on a chocolate agar plate (Oxoid). A diameter of $39 \mathrm{~mm}$ was measured and considered as susceptible according to the CLSI guidelines (Clinical and Laboratory Standards Institute, 2013, VET01-S2), identifiying the strain as Biovar I. No specific susceptibility breakpoints are currently defined by CLSI or EUCAST for this method for F. tularensis.

\section{Disease monitoring}

For surveillance reasons, one mouse housed adjacent was also examined pathologically, despite not showing any clinical signs. No typical lesions were found macroscopically and histologically. Culture of liver, spleen and kidney was negative for F. tularensis. Exposed zookeepers in the wildlife park showed no clinical signs.

\section{Diagnosis}

Disease: Tularemia

Etiology: Francisella tularensis ssp. holarctica, Biovar I Histopathological diagnosis: multifocal necrosis in liver, spleen and mesenteric lymph nodes

\section{Discussion}

Francisella tularensis is a highly infectious bacterial pathogen that causes tularemia in humans and animals. Lagomorphs and rodents are suggested to serve as nat- ural reservoir and ticks as vectors ${ }^{10}$. The species $F$. tularensis comprises two subspecies, namely tularensis (Type A strain) and holarctica (Type B strain) ${ }^{5}$. The more virulent subspecies tularensis is mostly found in Northern America, whereas bolarctica is reported all over the northern hemisphere, including Europe ${ }^{5}$. Furthermore, type B strains (bolarctica) can be classified into biovars: biovar I is naturally susceptible to erythromycin and occurs in Western Europe and North America, whereas biovar II is naturally resistant to erythromycin and occurs in Eastern Europe and Asia 5 .

The absence of gross organ lesions in the current case is rather unusual, according to pathological findings of 28 brown hares diagnosed with tularemia in Switzerland (collected 2012-2014). Necropsy of those animals revealed splenomegaly in all 28 animals, lesions of the tracheal mucosa in 25 and lymphadenomegaly in 15 animals ${ }^{7}$. Histopathology of the current case, however, showed typical lesions for tularemia, such as multiple necrotic foci in liver, spleen and lymph nodes, which correlated with the obtained positive bacteriological results.

The incidence of tularemia in humans in Switzerland increased significantly from seven cases in 2004 to 131 cases in 2017 according to the annual case numbers of the Swiss Federal Office for Public Health and is now considered a reemerging disease with an increasing veterinary public health relevance ${ }^{4,10}$. An epidemiological study of 276 human tularemia cases in Switzerland investigated causative risk factors for disease occurrence. $57 \%$ of the patients reported insect or arthropod bites (33\% tick-bite, $24 \%$ insects) and 22\% had contact with wild animals prior to contracting tularemia ${ }^{2,10}$. Recent studies in Northern Europe and Austria evaluated whether companion animals serve as a source of infection for humans, after contact to tularemia positive wild animals or contaminated environment. Both a Norwegian hunting $\operatorname{dog}$ and its owner contracted $F$. tularensis ssp. holarctica infection and developed tularemia after exposure to an infected mountain hare ${ }^{6}$. A subsequently launched post-exposition survey of 33 lemming hunting hounds revealed 13 seropositive dogs, whereof ten had suffered from nonspecific clinical signs ${ }^{6}$. A similar study in Austria reported five seropositive cases out of 80 tested hunting dogs 9 . Clinical cases of infected cats have only been reported from North America and only with the more virulent type A strains ${ }^{3}$. However, transmission from cats to humans has been described in North America as well as in Europe, where two people in Scandinavia contracted F. tularensis ssp. holarctica in- 
fection following a cat bite ${ }^{3,8,11}$. In contrast, 80 domestic cats living in tularemia endemic regions (Austria) were all serologically tested negative for F. tularensis ${ }^{9}$. A commercial vaccine is not available whether in human nor in veterinary medicine.

\section{References}

${ }_{1}$ Abril C, Nimmervoll H, Pilo P, Brodard I, Korczak B, Markus $\mathrm{S}$, et al.: Rapid diagnosis and quantification of Francisella tularensis in organs of naturally infected common squirrel monkeys (Saimiri sciureus). Vet Microbiol 2008: 127(1-2): 203-208.

2 Ehrensperger F, Riederer L, Friedl A: [Tularemia in a jogger woman after the attack by a common buzzard (Buteo buteo): A "One Health" case report]. Schweiz Arch Tierheilkd 2018: 160(3): 185-188.

${ }^{3}$ Larson MA, Fey PD, Hinrichs SH, Iwen PC: Francisella tularensis bacteria associated with feline tularemia in the United States. Emerg Infect Dis 2014: 20(12): 2068-2071.

${ }^{4}$ Lyko C, Chuard C: [Tularemia, an emerging disease in Switzerland]. Rev Med Suisse 2013: 9(401): 1816-1818, 1820.

${ }_{5}^{5}$ Maurin M, Gyuranecz M: Tularaemia: clinical aspects in Europe. Lancet Infect Dis 2016: 16(1): 113-124.

${ }_{6}$ Nordstoga A, Handeland K, Johansen TB, Iversen L, Gavier-Widen D, Mattsson R, et al.: Tularaemia in Norwegian dogs. Vet Microbiol 2014: 173(3-4): 318-322.
7 Origgi FC, Pilo P: Francisella tularensis Clades B.FTN002-00 and B.13 Are Associated With Distinct Pathology in the European Brown Hare (Lepus europaeus). Vet Pathol 2016: 53(6): 1220-1232.

8 Petersson E, Athlin S: Cat-bite-induced Francisella tularensis infection with a false-positive serological reaction for Bartonella quintana. JMM Case Rep 2017: 4(2): e005071.

9 Posautz A, Gyuranecz M, Denes B, Knauer F, Dier H, Walzer C: Seroprevalence of Francisella tularensis in Austrian Hunting Dogs. Vector Borne Zoonotic Dis 2018: 18(2): 117119.

10 Wittwer M, Altpeter E, Pilo P, Gygli SM, Beuret C, Foucault F, et al.: Population Genomics of Francisella tularensis subsp. holarctica and its Implication on the Eco-Epidemiology of Tularemia in Switzerland. Front Cell Infect Microbiol 2018: 8: 89.

11 Yaqub S, Bjornholt JV, Enger AE: [Tularemia from a cat bite]. Tidsskr Nor Laegeforen 2004: 124(24): 3197-3198.

\section{Corresponding author}

Sophie Peterhans

Institute of Veterinary Bacteriology

Vetsuisse Faculty, University of Zurich

Winterthurerstrasse 270

8057 Zurich, Switzerland

Phone: +41446358613

E-Mail: sophie.peterhans@uzh.ch
Case of the month: What's your diagnosis?

S. Peterhans et al. 